\title{
Effects of Neutrophil Migration Inhibitory Factors on Neonatal Neutrophils
}

\author{
INGEBORG C. KOWANKO, ANTONIO FERRANTE, AND GEORGE M. MAXWELL \\ University Department of Paediatrics and Department of Immunology, Adelaide Children's Hospital, North \\ Adelaide, South Australia
}

\begin{abstract}
Neonatal neutrophil migration was inhibited by preincubation with a lymphokine/monokine-rich medium conditioned by phytohemagglutinin-stimulated mononuclear leucocytes. Medium conditioned by unstimulated mononuclear cells or nonconditioned medium had no effect on neonatal neutrophil migration. Similar results were obtained with adult neutrophils. Migration distances in the presence and absence of a chemotactic gradient were much lower for neutrophils from neonates than adults when comparing treatments with the corresponding medium, i.e. medium conditioned by phytohemmaglutinin-stimulated mononuclear leucocytes, medium conditioned by unstimulated mononuclear leucocytes, or medium unconditioned by mononuclear leucocytes $(p<0.01)$. Although locomotion of both neonatal and adult neutrophils was inhibited by treatment, the percent inhibition of random migration was slightly but significantly less for neonates than adults ( $p$ $<0.05$ ). These results demonstrate that neutrophils from neonates are modulated by mononuclear leucocyte-derived mediators. (Pediatr Res 21: 377-380, 1987)
\end{abstract}

\section{Abbreviations}

MNL, mononuclear leucocytes

PHA, phytohemmaglutinin

sCM, medium conditioned by PHA-stimulated MNL nsCM, medium conditioned by unstimulated MNL

NCM, medium conditioned by MNL

FMLP, $N$-formyl-L-methionyl-L-leucyl-L-phenylalanine

Newborn infants, especially when born prematurely, are more susceptible to bacterial infections and experience greater morbidity and mortality from infections when compared to older children. The basis of the increased susceptibility of the neonate to infection is still not clear. Abnormalities of several components of the immune system of the neonate have been described and are believed to be contributing factors. Among these are findings that neonatal neutrophils are poor in chemotactic (1-7) and antimicrobial functions (8-14) compared with those of older children and adults.

There is evidence that mediators released by lymphocytes and monocytes (lymphokines and monokines) can augment the antimicrobial properties of neutrophils (15-20). Activation of neutrophils by these mediators is likely to occur during infection and may be essential for optimizing neutrophil microbial killing. We propose that neonatal neutrophils respond poorly to lym-

Received June 12, 1986; accepted November 21, 1986

Correspondence and reprint requests to Dr. I. Kowanko, University Department of Paediatrics and Department of Immunology, Adelaide Children's Hospital, North Adelaide, S.A., Australia 5006.

Supported by a grant from the Channel 10 Children's Medical Research Foundation. phokines and monokines and that this may be a contributing factor to increased susceptibility to infections in the neonate. In this study we examined the response of neonatal neutrophils to MNL-derived neutrophil migration inhibitory factors.

\section{MATERIALS AND METHODS}

Blood. Twenty-three umbilical cord blood samples from healthy term infants born by normal vaginal delivery and 16 venous blood samples from healthy adults were collected into tubes containing $125 \mathrm{U}$ lithium heparin. Informed consent was obtained from the mothers of the infants and from the adult volunteers. Neonatal and adult samples were prepared and tested in parallel on the same day.

Leucocyte separation. MNL and neutrophils were separated from adult blood by the rapid single-step method (21). The separation medium was prepared by mixing $17.4 \mathrm{ml}$ Angiografin, $11.3 \mathrm{ml}$ Urovist $\mathrm{S}, 8 \mathrm{~g}$ Ficoll, and $71.3 \mathrm{ml}$ distilled water. The density of this medium was brought to $1.114 \mathrm{~g} / \mathrm{ml}$ and $\mathrm{pH} 7.0$. A modified medium for isolating cord blood neutrophils was prepared by separately adjusting the densities of Angiografin and Urovist $\mathrm{S}$ to $1.114 \mathrm{~g} / \mathrm{ml}$ with $8 \%$ Ficoll, and then mixing equal volumes of these two solutions and adjusting the $\mathrm{pH}$ to 7.2. Angiografin (65\% aqueous meglumine amidotrizoate) and Urovist $\mathrm{S}$ (50\% aqueous sodium diatrizoate) were obtained from Schering AG (Berlin/Bergkamen, Germany) and Ficoll (mol wt $=400,000$ ) was obtained from Pharmacia.

Blood was layered onto the appropriate separation medium and centrifuged at $500 \times g$ for $30 \mathrm{~min}$. MNL collected as a band at the plasma-medium interface and neutrophils collected as a second distinct band. Occasionally erythrocyte contamination of the neonatal neutrophil band was observed and in these cases the red blood cells were lysed by incubating with $0.87 \%$ ammonium chloride for $5 \mathrm{~min}$ at $37^{\circ} \mathrm{C}$. Neutrophils were washed three times in medium 199 (Commonwealth Serum Laboratories, Melbourne, Australia) and resuspended at $4 \times 10^{7} / \mathrm{ml}$.

Preparation of $M N L$-conditioned media. MNL-conditioned medium was prepared as described previously $(15,16)$. Briefly, adult MNL were cultured in the presence or absence of $1 \mu \mathrm{g} / \mathrm{ml}$ PHA in RPMI 1640 tissue culture medium (Flow Laboratories, Sydney, Australia) containing 2.5\% human AB serum for $24 \mathrm{~h}$, washed, and recultured for another $48 \mathrm{~h}$. The supernatants were centrifuged to remove cells and debris, millipore filtered $(0.22$ $\mu \mathrm{m}$ ), and stored in aliquots at $-20^{\circ} \mathrm{C}$. Medium conditioned by PHA-stimulated MNL was termed sCM. Medium conditioned by MNL without PHA (nsCM) and medium unconditioned by MNL (NCM) were used as controls. The same batch of conditioned media was used throughout this study. SCM is known to contain a mixture of lymphokines and monokines including interleukin-2, colony-stimulating factors, neutrophil migration inhibitory factors, and factors that enhance neutrophil amebicidal, bactericidal, and tumoricidal activity and induce a respiratory burst. These factors are undetectable in nsCM and NCM.

Treatment of neutrophils with conditioned media. One volume 
of cell suspension and three volumes of conditioned media were mixed to produce a final concentration of $10^{7}$ cells $/ \mathrm{ml}$ and incubated at $37^{\circ} \mathrm{C}$ in a humidified $5 \% \mathrm{CO}_{2}$-air atmosphere for $1 \mathrm{~h}$. For each cord blood sample or adult sample separate incubations of neutrophils with $\mathrm{sCM}$, nsCM, and $\mathrm{NCM}$ were performed simultaneously. After incubation cells were centrifuged and resuspended at $4 \times 10^{7} / \mathrm{ml}$ in medium 199 .

Neutrophil chemotaxis and random movement. Neutrophil random mobility and chemotactic responsiveness under agarose were measured as previously described $(22,23)$. Agarose plates were prepared by mixing $3 \mathrm{ml}$ of $2 \%$ agarose with $3 \mathrm{ml}$ of double strength medium 199 containing $10 \%$ heat inactivated fetal calf serum. Wells $2.5 \mathrm{~mm}$ in diameter were cut with the aid of a template. For chemotaxis, three wells in a straight line were cut $2.5 \mathrm{~mm}$ apart. Five $\mu \mathrm{l}$ cell suspension $\left(2 \times 10^{6}\right.$ cells $)$ were added to the center well and $5 \mu$ lof the chemotactic agent $10^{-7} \mathrm{M}$ FMLP or dimethyl sulphoxide control added to the outer wells. The plates were incubated for $90 \mathrm{~min}$ at $37^{\circ} \mathrm{C}$ in a humidified $5 \% \mathrm{CO}_{2}$-air atmosphere. Chemotactic migration measured with an inverted microscope using an eyepiece grid, was taken as the distance traveled by the 10 fastest moving cells in the direction of the well containing FMLP. Random migration distance was similarly mcasured as the mean distance traveled in four directions by the 10 fastest moving cells from wells in the absence of a chemotactic gradient. Duplicate wells on duplicate plates were set up for each sample for both random and chemotactic migrations.

Statistics. Random and chemotactic migration distances of neonatal and adult neutrophils preincubated with $\mathrm{sCM}$, nsCM, and NCM were compared. The migration inhibition effect of the sCM was calculated as the difference in migration distance between sCM- and nsCM-preincubated neutrophils as a percentage of the nsCM - preincubated neutrophil migration distance. Wilcoxon's matched pairs signed rank tests were used to compare mean migration distances of neonate and adult neutrophils prepared and tested on the same day.

\section{RESULTS}

The data for random and chemotactic migration distances of neonatal and adult neutrophils preincubated with corresponding media are presented in Tables 1 and 2 . The percent inhibition of migration due to $\mathrm{SCM}$ and the results of the paired nonparametric statistical comparisons between neonatal and adult neutrophil migration are also included in Tables 1 and 2.

Neonatal neutrophils show significantly decreased random and chemotactic migration compared to adult neutrophils preincubated with corresponding media. Preincubation with $\mathrm{SCM}$ inhibited both random and chemotactic migration of neonatal and adult neutrophils. The percent inhibition caused by $\mathrm{sCM}$ of random migration was significantly lower for neonatal neutrophils compared to adult neutrophils but there was no significant difference in percent inhibition due to $\mathrm{SCM}$ of chemotactic migration of neonatal and adult neutrophils.

The data presented show that there were wide variations in migration distances of the neonatal neutrophils within treatment groups. We considered the time elapsed between collection and separation of the blood $(1-12 \mathrm{~h})$, duration of centrifugation

Table 1. Effects of neutrophil migration inhibitory factors on neonatal neutrophil random migration

\begin{tabular}{|c|c|c|c|c|c|c|c|c|}
\hline & \multicolumn{6}{|c|}{ Random migration distance $(\mathrm{mm})^{*}$} & \multirow{2}{*}{\multicolumn{2}{|c|}{$\begin{array}{l}\% \text { inhibition } \\
\text { due to sCM* }\end{array}$}} \\
\hline & \multicolumn{2}{|c|}{$\mathrm{sCM}$} & \multicolumn{2}{|c|}{$\mathrm{nsCM}$} & \multicolumn{2}{|c|}{$\mathrm{NCM}$} & & \\
\hline & Adult & Neonate & Adult & Neonate & Adult & Neonate & Adult & Neonate \\
\hline & 0.38 & 0.15 & 0.61 & 0.42 & 0.66 & 0.44 & 38 & 63 \\
\hline & 0.25 & 0.13 & 0.66 & 0.38 & 0.66 & 0.44 & 61 & 65 \\
\hline & 0.12 & $0.12 \dagger$ & 0.38 & $0.36 \dagger$ & 0.40 & $0.37 \dagger$ & 67 & $64 \dagger$ \\
\hline & 0.12 & $0.13 \dagger$ & 0.38 & $0.42 \dagger$ & 0.40 & $0.44 \dagger$ & 67 & $68 \dagger$ \\
\hline & 0.34 & 0.38 & 0.64 & 0.66 & 0.62 & 0.58 & 47 & 43 \\
\hline & 0.34 & 0.34 & 0.64 & 0.62 & 0.62 & 1.00 & 47 & 45 \\
\hline & 0.41 & 0.24 & 0.47 & 0.30 & 0.60 & 0.41 & 12 & 21 \\
\hline & 0.50 & 0.32 & 0.62 & 0.37 & 0.87 & 0.48 & 19 & 14 \\
\hline & 0.26 & $0.18 \dagger$ & 0.41 & $0.60 \dagger$ & 0.65 & $0.53 \dagger$ & 35 & $68 \dagger$ \\
\hline & 0.26 & $0.14 \dagger$ & 0.42 & $0.20 \dagger$ & 0.42 & $0.26 \dagger$ & 36 & $30 \dagger$ \\
\hline & 0.26 & $0.08+$ & 0.42 & $0.11 \dagger$ & 0.42 & $0.18 \dagger$ & 36 & $23 \dagger$ \\
\hline & 0.23 & 0.26 & 0.63 & 0.40 & 0.51 & 0.34 & 63 & 34 \\
\hline & 0.19 & 0.16 & 0.50 & 0.22 & 0.54 & 0.20 & 61 & 24 \\
\hline & 0.19 & 0.17 & 0.50 & 0.36 & 0.54 & 0.36 & 61 & 52 \\
\hline & 0.28 & 0.10 & 0.60 & 0.16 & 0.56 & 0.23 & 50 & 40 \\
\hline & 0.28 & 0.10 & 0.60 & 0.18 & 0.56 & 0.22 & 50 & 46 \\
\hline & 0.34 & 0.22 & 0.64 & 0.44 & 0.55 & 0.48 & 46 & 50 \\
\hline & 0.23 & 0.16 & 0.42 & 0.24 & 0.45 & 0.30 & 44 & 30 \\
\hline & 0.12 & 0.18 & 0.32 & 0.36 & 0.48 & 0.49 & 63 & 49 \\
\hline & 0.12 & $0.16 \dagger$ & 0.32 & $0.20 \dagger$ & 0.48 & $0.05 \dagger$ & 63 & $19 \dagger$ \\
\hline & 0.80 & 0.64 & 0.88 & 0.87 & 0.93 & 0.78 & 9 & 25 \\
\hline & 0.19 & 0.22 & 0.64 & 0.53 & 0.66 & 0.54 & 69 & 37 \\
\hline & 0.19 & 0.15 & 0.64 & 0.25 & 0.66 & 0.32 & 69 & 39 \\
\hline$n$ & 16 & 23 & 16 & 23 & 16 & 23 & 16 & 23 \\
\hline Median & 0.26 & 0.16 & 0.60 & 0.36 & 56 & 0.37 & 46 & 39 \\
\hline Mean & 0.30 & 0.20 & 0.55 & 0.37 & 0.60 & 0.39 & 43 & 41 \\
\hline $\mathrm{SD}$ & 0.16 & 0.12 & 0.14 & 0.18 & 0.14 & 0.17 & 20 & 16 \\
\hline$p$ & \multicolumn{2}{|c|}{$<0.01$} & \multicolumn{2}{|c|}{$<0.01$} & \multicolumn{2}{|c|}{$<0.01$} & \multicolumn{2}{|c|}{$<0.05$} \\
\hline
\end{tabular}

* Random migration and percent inhibition for neutrophils from each neonate and adult after preincubation with conditioned media. Data on each horizontal row were obtained on the same day. $p=$ probability of statistically significant difference between neonate and adult using Wilcoxon's paired test.

$\dagger$ Neonatal blood samples requiring both hypotonic lysis and more than 50 min centrifugation. 
Table 2. Effect of neutrophil migration inhibitory factors on neonatal neutrophil chemotaxis

\begin{tabular}{|c|c|c|c|c|c|c|c|c|}
\hline & \multicolumn{6}{|c|}{ Chemotactic migration distance $(\mathrm{mm})^{*}$} & \multirow{2}{*}{\multicolumn{2}{|c|}{$\begin{array}{l}\% \text { inhibition } \\
\text { due to SCM* }\end{array}$}} \\
\hline & \multicolumn{2}{|c|}{$\mathrm{sCM}$} & \multicolumn{2}{|c|}{$\mathrm{nsCM}$} & \multicolumn{2}{|c|}{$\mathrm{NCM}$} & & \\
\hline & Adult & Neonate & Adult & Neonate & Adult & Neonate & Adult & Neonate \\
\hline & 1.00 & 1.06 & 1.76 & 1.20 & 1.50 & 0.86 & 43 & 11 \\
\hline & 1.30 & 0.46 & 1.66 & 0.90 & & & 22 & 48 \\
\hline & 0.40 & $0.30 \dagger$ & 1.81 & $1.13 \dagger$ & 1.91 & $1.28 \dagger$ & 77 & $73 \dagger$ \\
\hline & 0.40 & $0.36 \dagger$ & 1.81 & $1.63 \dagger$ & 1.91 & $1.76+$ & 77 & $77 \dagger$ \\
\hline & 1.66 & 1.60 & 2.16 & 1.90 & 1.93 & 1.46 & 23 & 15 \\
\hline & 1.66 & 1.30 & 2.16 & 1.43 & 1.93 & 1.80 & 23 & 9 \\
\hline & 1.83 & 1.30 & 1.96 & 1.43 & 2.10 & 1.60 & 6 & 11 \\
\hline & 1.03 & 0.40 & 1.66 & 0.56 & 1.76 & 0.70 & 38 & 29 \\
\hline & 1.60 & $0.76 \dagger$ & 2.26 & $1.46 \dagger$ & 2.33 & $1.40 \dagger$ & 29 & $47 \dagger$ \\
\hline & 1.60 & 0.82 & 2.26 & 1.55 & 2.33 & 1.85 & 29 & 47 \\
\hline & 1.94 & 0.48 & 2.38 & 0.50 & 1.90 & 0.46 & 18 & 4 \\
\hline & 1.60 & 0.86 & 2.00 & 1.56 & 2.00 & 1.63 & 20 & 44 \\
\hline & 1.33 & 0.40 & 2.00 & 1.53 & 2.53 & 1.46 & 33 & 73 \\
\hline & 2.23 & 1.76 & 2.26 & 2.26 & 2.53 & 2.13 & 1 & 22 \\
\hline & 0.86 & 0.68 & 2.10 & 1.84 & 2.30 & 1.91 & 58 & 63 \\
\hline & 0.86 & 0.83 & 2.10 & 1.08 & 2.30 & 1.14 & 58 & 22 \\
\hline$n$ & 12 & 16 & 12 & 16 & 11 & 15 & 12 & 16 \\
\hline Mean & 1.40 & 0.82 & 2.00 & 1.34 & 2.00 & 1.38 & 29 & 34 \\
\hline SD & 0.51 & 0.46 & 0.24 & 0.46 & 0.38 & 0.45 & 21 & 25 \\
\hline$p$ & \multicolumn{2}{|c|}{$<0.01$} & \multicolumn{2}{|c|}{$<0.01$} & \multicolumn{2}{|c|}{$<0.01$} & \multicolumn{2}{|c|}{ NS } \\
\hline
\end{tabular}

* Chemotactic migration and percent inhibition for neutrophils from each neonate and adult after preincubation with conditioned media. Data on each horizontal row were obtained on the same day. $p=$ probability of statistically significant difference between neonate and adults using Wilcoxon's paired test.

$\uparrow$ Neonatal blood samples requiring both hypotonic lysis and more than 50 min centrifugation.

through the density gradient, and erythrocyte lysis as possible sources of variation. It was found that cord blood samples which required more than $50 \mathrm{~min}$ centrifugation through the gradient as well as erythrocyte lysis showed decreased random and chemotactic neutrophil migration compared to samples requiring either long centrifugation or lysis alone. There was no significant effect of the time elapsed between collection and separation of the blood sample on migration distance.

\section{DISCUSSION}

Our results show that both random and chemotactic migration of neonatal neutrophils was impaired compared to adult cells. Neonatal neutrophils migrate only about $60 \%$ of the distance that adult neutrophils move over a 90 -min period. The reduced chemotactic migration of neonatal neutrophils has been well documented $(1-16)$ but there have been limited studies on random movement. Our demonstration of decreased random mobility of neutrophils from newborn babies confirms the results of Zeligs et al. (7), but contrasts with those of Tono-Aka et al. (1) who used a more complicated method of leucocyte separation.

It has been suggested that the differences between adult and newborn neutrophil chemotactically directed migration may be related to decreased availability of receptors for chemotactic factors $(2,24-26)$. Defects in the neonatal neutrophil membrane and cytoskeletal elements have also been implicated as possible reasons for their decreased mobility. For example, newborn neutrophils exhibit reduced deformability (2), irreversible aggregation (25), and reduced Concanavalin A capping (27,28), all of which reflect abnormalities in the membrane. Cord blood neutrophils may include more immature forms than adult cells (29), or may represent different proportions of cells deriving from circulating or marginated pools. It is not known whether these differences are functionally important.

We have previously shown that $\mathrm{SCM}$ contains neutrophil migration inhibitory factor(s) active on adult neutrophils which might be leucocyte inhibitory factor (20). This study provides the first evidence that neonatal neutrophils also respond to this factor which causes a $40 \%$ decrease in random migration distance. However, paired statistical tests between adults and neonates suggest that the percent inhibition of random migration is significantly less in neonates than in adults. An impaired response to migration inhibitory factors could result in less efficient retention of neonatal neutrophils at the site of infection, and consequently contribute to the impaired response of neonates to bacterial infections. Chemotactic migration of adult and neonatal neutrophils was also inhibited by SCM by about $30 \%$ but there was no significant difference in the degree of inhibition between adults and newborns. This may be related to differences in mechanisms and controls of random and chemotactic movements (23).

Neutrophils are important cells dealing with bacterial infections of the types causing problems in neonates. Defects in their function may be critical in tipping the balance between successful resolution of infection or progression to overwhelming bacteremia. There is evidence that neonatal neutrophils may have abnormalities in their oxidative metabolism compared to adult cells (8-13) and that bactericidal activity of neonates may be impaired (14). sCM is known to contain lymphokine factors that modulate these neutrophil functions $(15-20)$. The results of this investigation suggest that neonatal neutrophils may show a reduced response to MNL-derived migration inhibitory factors. Further studies are needed to determine whether neonatal neutrophils might be less competent to respond to an array of MNLderived factors than adult cells.

Acknowledgment. The authors thank the staff of the labor wards at the Queen Victoria Hospital and Flinders Medical Centre who collected the cord blood samples.

\section{REFERENCES}

1. Tono-Aka T, Nakayama M, Uehara H, Matsumoto S 1979 Characteristics of impaired chemotactic function in cord blood leukocytes. Pediatr Res 13:148151 
2. Mease AD, Fischer GW, Hunter KW, Ruymann FB 1980 Decreased phytohcmagglutinin-induced aggregation and C5a-induced chemotaxis of human newborn neutrophils. Pediatr Res 14:142-146

3. Yegin O 1983 Chemotaxis in childhood. Pediatr Res 17:183-187

4. Klein RB, Fischer TJ, Gard SE, Biberstein M, Rich KC, Stiehm RS 1977 Decreased mononuclear and polymorphonuclear chemotaxis in human newborns, infants and young children. Pediatrics 60:467-472

5. Sacchi F, Rondini G, Mingrat G, Stronati M, Gancia GP, Marseglia GL Siccari AG 1982 Different maturation of neutrophil chemotaxis in term and preterm infants. J Pediatr 101:273-274

6. Sacchi F, Hill HR 1984 Defective membrane potential changes in neutrophils from human neonates. J Exp Med 160:1247-1252

7. Zeligs BJ, Roberts R, Wientzen RL, Bellanti JA 1983 Decreased chemotactic and bactericidal activity of the neonatal neutrophil using a new method of cell separation. Pediatr Res 17:263A

8. Shigeoka AO, Santos JI, Hill HR 1979 Functional analysis of neutrophil granulocytes from healthy, infected and stressed neonates. J Pediatr 95:454 460

9. Ambruso DR, Altenburger KM, Johnston RB 1979 Defective oxidative metabolism in newborn neutrophils: discrepancy between superoxide anion and hydroxyl radical gencration. Pediatrics 64:722-725

10. Ambruso DR, Bentwood B, Henson PM, Johnston RB 1984 Oxidative metabolism of cord blood neutrophils: relationship to content and degranulation of cytoplasmic granules. Pediatr Res 18:1148-1153

11. Frazicr JP, Cleary TG, Pickering LK, Kohl S, Ross PJ 1982 Leucocyte function in healthy neonates following vaginal and cesarian section deliveries. J Pediatr 101:269-272

12. Strauss RG, Snyder EL 1983 Activation and activity of the superoxide generating system of neutrophils from human infants. Pediatr Res 17:662-664

13. Ambruso DR, Stork LC, Gibson BG 1985 Increased activity of the respiratory burst in cord blood neutrophils: kinetics of NADPH oxidase in subcellular fractions. Pediatr Res 19:275A

14. Stroobant J, Harris MC, Cody CS, Polin RA, Douglas SD 1984 Diminished bactericidal capacity for group B Streptococcus in neutrophils from "stressed" and healthy neonates. Pediatr Res 18:634-637

15. Ferrante A, Mocatta TJ 1984 Human neutrophils require activation by mononuclear leukocyte conditioned medium to kill the pathogenic free-living amoeba Naegleria fowleri. Clin Exp Immunol 56:559-566

16. Ferrante A, Abell TJ 1986 Conditioned medium from stimulated mononuclear leukocytes augments human neutrophil-mediated killing of a virulent Acan- thamoeba sp. Infect Immun 51:607-617

17. Pennington JE, Rossing TH, Boerth LW, Lee TH 1985 Isolation and partial characterization of a human alveolar macrophage-derived neutrophil activating factor. J Clin Invest 75:1230-1237

18. Inoue T, Sendo F 1983 In vitro introduction of cytotoxic polymorphonuclear leukocytes by supernatant from a concanavalin A-stimulated spleen cell culture. J Immunol 131:2508-2514

19. Lomnitzer R, Glover A, Rabson AR 1977 The effect of PHA-activated MNcell supernates on polymorphonuclear leukocyte function. Clin Exp Immunol 29:501-508

20. Klempner MS, Rocklin RE 1983 Effects of leucocyte inhibitory factor (LIF) on human neutrophil function. Inflammation 7:145-153

21. Ferrante A, Thong YH 1978 A rapid one-step procedure for purification of mononuclear and polymorphonuclear leukocytes from human blood using a modification of the hypaque-ficoll method. J Immunol Methods 24:389393

22. Nelson RD, Quie PG, Simmons RL 1975 Chemotaxis under agarose: a new and simple method for measuring chemotaxis and spontaneous migration of human polymorphonuclear leukocytes and monocytes. J Immunol 115:1650-1656

23. Ferrante A, Beard LJ, Thong YL 1980 Early decay of human neutrophil chemotactic responsiveness following isolation from peripheral blood. Clin Exp Immunol 39:532-537

24. Nunoi H, Endo F, Chikazawa S, Namikawa T, Matsuda I 1983 Chemotactic receptor of cord blood granulocytes to the synthesized chemotactic peptide $N$-formyl-methionyl-leucyl-phenylaniline. Pediatr Res 17:57-60

25. Mease AD, Burgess DP, Thomas PJ 1981 Irreversible neutrophil aggregation. A mechanism of decreased newborn neutrophil chemotactic response. Am J Pathol 104:98-102

26. Anderson DC, Hughes BJ, Wayne-Smith C 1981 Abnormal mobility of neonatal polymorphonuclear leukocytes. J Clin Invest 68:863-874

27. Kimura GM, Miller ME, Leake RD, Raghunathan R, Cheung ATW 1981 Reduced concanavalin A capping of neonatal polymorphonuclear leucocytes. Pediatr Res 15:1271-1273

28. Strauss RG, Hart MJ 1981 Spontaneous and drug-induced concanavalin A capping of neutrophils from human infants and their mothers. Pediatr Res 15:1314-1318

29. Krause PJ, Kosciol C, Pontius LT, Malech HL 1985 Neutrophil heterogeneity in neonates and adults. Pediatr Res 19:277A 